\title{
Descriptive Epidemiology for Mycoplasma pneumoniae Infection Using (Nursery) School Absenteeism Surveillance System, and Proposal for Countermeasures
}

\author{
Junko Kurita', Natsuki Nagasu², Noriko Nagata ${ }^{3}$, Naomi Sakurai ${ }^{4}$, Yasushi Ohkusa ${ }^{5}$, \\ Tamie Sugawara ${ }^{5}$
}

${ }^{1}$ The Graduate School of Health Sciences, Ibaraki Prefectural University, Ibaraki, Japan

${ }^{2}$ Health Services Disease Control Division, Department of Health and Social Services, Ibaraki, Japan

${ }^{3}$ Ibaraki Prefectural Institute of Public Health, Ibaraki, Japan

${ }^{4}$ Center for Medical Sciences, Ibaraki Prefectural University of Health Sciences, Ibaraki, Japan

${ }^{5}$ National Institute of Infectious Diseases, Tokyo, Japan

Email: tammy@nih.go.jp

How to cite this paper: Kurita, J., Nagasu, N., Nagata, N., Sakurai, N., Ohkusa, Y. and Sugawara, T. (2018) Descriptive Epidemiology for Mycoplasma pneumoniae Infection Using (Nursery) School Absenteeism Surveillance System, and Proposal for Countermeasures. Journal of Biosciences and Medicines, 6, 33-42.

https://doi.org/10.4236/jbm.2018.610005

Received: June 18, 2018

Accepted: October 9, 2018

Published: October 12, 2018

Copyright $\odot 2018$ by authors and Scientific Research Publishing Inc. This work is licensed under the Creative Commons Attribution International License (CC BY 4.0).

http://creativecommons.org/licenses/by/4.0/

\section{(c) (i) Open Access}

\begin{abstract}
Objective: We present descriptive epidemiology of Mycoplasma infection using the (Nursery) School Absenteeism Surveillance System ((N)SASSy) in addition to national official sentinel surveillance for infectious diseases (NOSSID). It is expected to be helpful for early detection and response to outbreak of Mycoplasma infection and also to be useful for antimicrobial resistance measures. Method: The study period was 2010-2014 seasons. The study area was the whole of Ibaraki prefecture, Japan. NOSSID reports the number of patients with Mycoplasma pneumonia from 13 sentinel hospitals. We compare the number of patients and the incidence rate of NOSSID with the same information in (N)SASSy. Result: In NOSSID, the largest number of patients by age was 27 patients of one year old in 2012. (N)SASSy showed that the incidence rate in the elementary schools is high in 2011 and 2012. Especially, the second grade students were the highest. Discussion: Even though $M y$ coplasma infection is well known as a pediatric disease, we showed that second grade was the highest in incidence at first. Because (N)SASSy is timely and real-time information collection, evaluation and sharing countermeasures with (nursery) schools, public health centers, and physicians are useful for students and nursery school children, and communities. Conclusion: Because mycoplasma infection is common pediatric infectious diseases and because some patients rarely develop severe infections, we must prevent larger outbreaks. (N)SASSy can provide timely intervention at the initial phase of
\end{abstract}


outbreak by monitoring situations in (nursery) schools and comparing data to baseline information.

\section{Keywords}

Mycoplasma Infection, (Nursery) School Absenteeism Surveillance System, Surveillance

\section{Introduction}

Mycoplasma pneumoniae infection is a respiratory infectious disease caused by Mycoplasma pneumoniae. Its symptoms are stubborn cough, fever, worthless feeling, and headache. It sometimes develops to pneumonia, Mycoplasma pneumoniae pneumonia, and hospitalization. Incidence of Mycoplasma pneumoniae pneumonia is well known to be higher in 3 - 15-year-old children: it accounts for $7 \%-30 \%$ of community-acquired pneumonia [1]. It's also known for its activity throughout the year, with winter being a season of slightly higher incidence. Moreover, outbreaks occur at $4-7$ year intervals [1]. Especially in Japan, the interval is said to be about four years [2].

In Japan, National Official Sentinel Surveillance for Infectious Disease (NOSSID) based on the Infection Control Law requires sentinel hospitals to report cases of Mycoplasma pneumoniae pneumonia. Sentinels were selected as hospitals with more than 300 beds which provide pediatric and internal medicine, with more than one hospital per secondary medical zone. In Ibaraki prefecture, 13 hospitals are designated as sentinel hospitals: almost $1 \%$ of 1400 hospital/clinics for the entire 2.9 million populations. Therefore, NOSSID cannot capture moderate cases for which patients are not hospitalized or hospitalized patients, even severe cases, at non-sentinel hospitals.

NOSSID revealed that large outbreaks of Mycoplasma pneumoniae pneumonia occurred before 1990 in Japan. However, such large outbreaks have ceased recently [3]. Since 2000, the number of patients per sentinel had been increasing gradually. For example in Ibaraki prefecture, remarkable outbreaks occurred in 2011 and 2012.

Students or nursery schoolchildren are sometimes absent from (nursery) school because of Mycoplasma pneumoniae infection. Hereinafter, we designate both nursery schools and schools as (nursery) schools for brevity. We can observe their dynamics using the (Nursery) School Absenteeism Surveillance System ((N)SASSy). Actually, (N)SASSy uses Japan's own unique at (nursery) school culture. The family of a student who is absent from a (nursery) school because of infectious diseases usually reports that fact to the (nursery) school. School nurses and other teachers provide information to (N)SASSy via the internet. As of the end of 2016, the system covered approximately 37,000 schools, including 10,000 nursery schools, or about $60 \%$ of all schools and $40 \%$ of all nursery schools in Japan. On a daily basis, it monitors the health conditions of 
about four million children younger than 18 years old. The (N)SASSy information is used by (nursery) schools themselves to recognize situations in surrounding areas and to inform teachers, students, and caregivers, thereby promoting precautionary measures if some outbreaks is found in surrounding areas but not at their own (nursery) school. The (N)SASSy information is also shared among (nursery) school doctors, educational boards, local government offices for nursery schools, public health centers, and local medical associations. That shared information encourages earlier awareness of infectious diseases, which engenders earlier responses to the initial stage of outbreaks in (nursery) schools. Moreover, having such a large trove of data related to children's health facilitates large epidemiological studies. It has been developed by the research group headed by Dr. Ohkusa, one of the authors of this paper, funded by the Ministry of Health, Labour and Welfare since 2007 and has been keeping its copyright [4] [5]. Currently, the system is operated by the Japanese Society of School Health.

In Ibaraki prefecture, all schools started to participate in (N)SASSy in 2009. All nursery schools started to use the system in 2012 [6] [7]. In 2014, it became possible to monitor the health condition of all children in (nursery) schools from zero to eighteen years old in a timely manner. This report describes the dynamics of Mycoplasma pneumoniae infection in (nursery) schoolchildren using (N)SASSy and compares them with NOSSID. Then we present consideration of how to remind the public health organizations, staff of (nursery) schools and caregivers about precautions against Mycoplasma pneumoniae infection.

Resistance of Mycoplasma pneumonia against antibiotics has been increasing. In Ibaraki prefecture, about half of the tested cases showed resistance to macrolide antibiotics [8]. Therefore, descriptive epidemiology of Mycoplasma pneumoniae infection is also necessary for antimicrobial resistance measures.

\section{Methods}

We define Mycoplasma pneumoniae infection as an illness caused by Mycoplasma pneumoniae. Mycoplasma pneumoniae pneumonia is pneumonia caused by Mycoplasma pneumoniae. NOSSID monitors Mycoplasma pneumoniae pneumonia and (N)SASSy records patients with Mycoplasma pneumoniae infection.

The study period was April 2010 - March 2014. The study area was the whole of Ibaraki prefecture.

Although NOSSID reports the number of patients with infection disease by epidemiological weeks, (N)SASSy records the number of absences daily in the school year. To compare the two data, NOSSID data are divided as the $14^{\text {th }}$ epidemiological week to the $13^{\text {th }}$ epidemiological week in subsequent years to accommodate the definition of the study period. NOSSID reports the number of Mycoplasma pneumoniae pneumonia patients from 13 sentinel hospitals.

Mycoplasma pneumoniae infection was classified as the third group of "other infectious diseases" in the School Health and Safety Act defined as not a minor infection, but one which has a high probability of spreading in situations of 
group life in school. Although students with the first or second group under the Law cannot attend schools when "suspended due to infectious diseases", students with the third group can sometimes be regarded as "suspended due to infectious diseases" but can sometimes be judged as not "suspended due to infectious diseases" by the school principal. Therefore, patients with Mycoplasma pneumoniae infection are not necessarily "suspended due to infectious diseases", even if they were diagnosed as having Mycoplasma pneumoniae infection. In fact, (N)SASSy records not only "suspended due to infectious diseases", but also those who were not applied as "suspended due to infectious diseases" if they were so diagnosed.

As of May, 2014 in Ibaraki prefecture, (N)SASSy covers 523 nursery schools with 48 thousand children in 1260 kindergarten, elementary, junior high and high schools, with 377 thousand students including private schools. The proportion of data entered into (N)SASSy among (nursery) schools was $70 \%-80 \%$; its average was about 76\% during 19 - 23 January, 2015.

We compared the number of patients in NOSSID and the incidence rate, defined as the number of patients divided by enrollment by age in (N)SASSy, with comparable information in (N)SASSy. We also compared the number of patients youger than 18 years old in NOSSID and in (N)SASSy.

\section{Ethical Considerations}

No (N)SASSy information was personally identifiable information such as a name, addresses, or date of birth. Moreover, we counted only patients with a certain condition from the collected information. Ethical guidelines for epidemiological research in Japan require no receipt of informed consent from patients in this case. Information from NOSSID was published data, in general.

\section{Results}

Figure 1 depicts the number of patients per sentinel in NOSSID. The highest number of patient per sentinel was 1.31 , recorded in the $42^{\text {nd }}$ week in 2010 , and the $30^{\text {th }}, 44^{\text {th }}$, and $45^{\text {th }}$ weeks in 2012. In 2011, the highest was 0.92 in the $42^{\text {nd }}$ week; it was also 0.92 in the $21^{\text {st }}$ week in 2013. In 2014, it was 0.46 in the third week. For patients under 18 years old, the highest number per sentinel was 1.0 in the $34^{\text {th }}$ and $44^{\text {th }}$ epidemiological weeks in 2012.

Figure 2 depicts the number of patients by age in NOSSID. The proportions of patients younger than 18 years old were $33.7 \%, 36.9 \%, 70.6 \%, 84.4 \%$, and $94.7 \%$, respectively, in 2010-2014. The largest number of patients by age was 27 patients of one year old in 2012, followed by 25 patients of $4-5$ years old in the same year.

Figure 3 depicts the epidemic curve of Mycoplasma pneumoniae infection in (N)SASSy. Although the highest number of patients per day in the first term during April - July in 2011 was around 40, it was higher than 40 in the second 


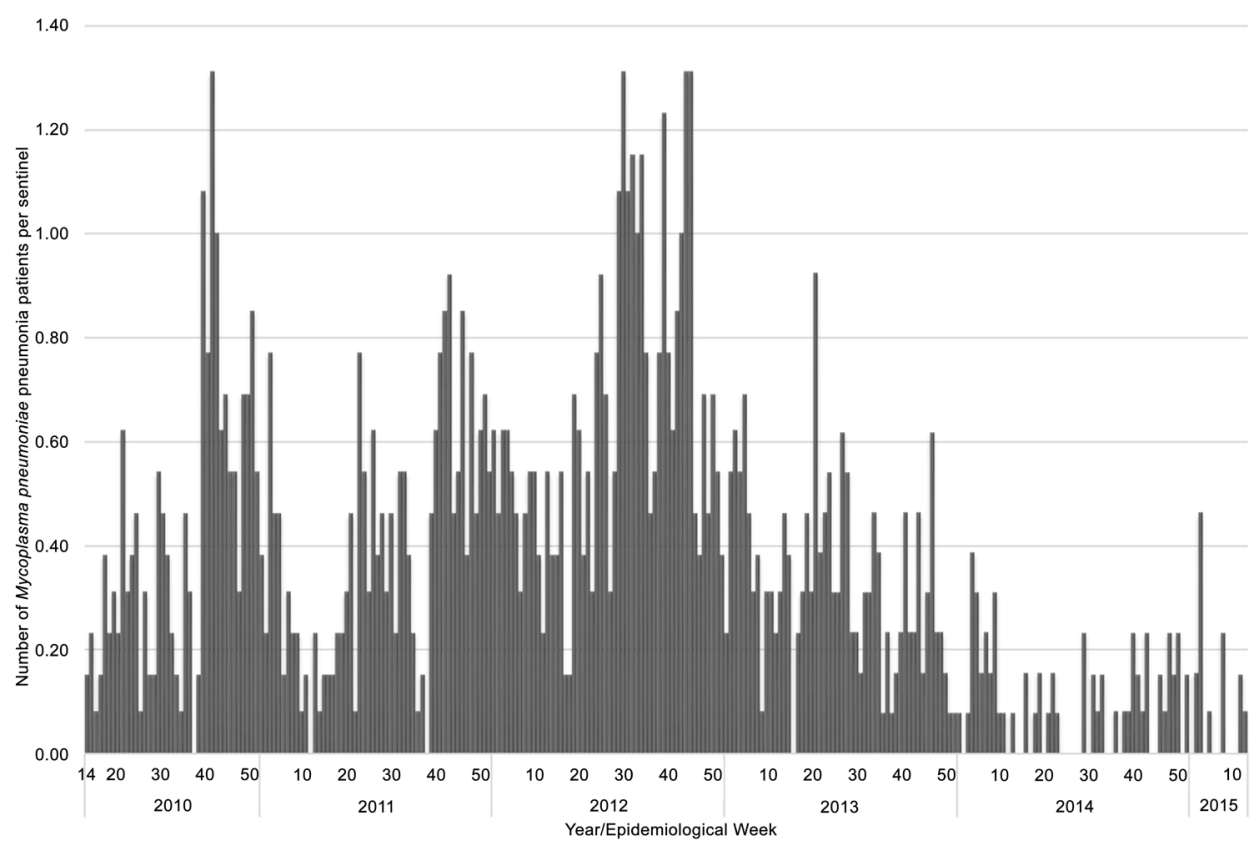

Figure 1. Number of Mycoplasma pneumoniae pneumonia patients per sentinel in Ibaraki prefecture in NOSSID. Note: This figure the number of newly diagnosed patients since 2010 school year to 2014 school year epidemiological week by week per sentinel. Because the school year in Japan is defined as the first of April extending to the end of March of the following year, we defined the school year in this figure as the $14^{\text {th }}$ epidemiological week to the 13 rd epidemiological week of the following year. Epidemiological week in Japan are defined as Monday through Sunday. The first epidemiological week is a week including the first of January, if the day of the week of the first of January was Monday-Wednesday. If the day of the week of the first of January was Thursday-Saturday, then the first epidemiological week starts from the first Monday in the year. Newly diagnosed patient were recorded in a week including the day when they were diagnosed as having Mycoplasma pneumonia. The number of sentinel in Ibaraki was 13.

term of September - December. During the 2012 season, it was gradually increasing. The highest number of patients in the study period, 119, was reached on December 26, 2012.

Figure 4 depicts the incidence rate by age in (N)SASSy. The incidence rates in the elementary schools, with children aged 6 - 11, were high in 2011 and 2012. Especially, the second grade, age 7, was the highest. Following were the third grade, age 8 , and the first grade, age 6 . Although NOSSID reported no patients of age 0 in 2011, 2012, and 2014 season, (N)SASSy reported respective incidence rates of age 0 as $0.36 \%, 1.69 \%$, and $1.19 \%$.

Table 1 presents a comparison of the number of patients younger than eighteen years old among the number of patients with Mycoplasma pneumoniae pneumonia and those younger than eighteen years in NOSSID and Mycoplasma pneumoniae infection in (N)SASSy. The NOSSID cover rate to (N)SASSy were $4.9,2.4,4.2,6.2$ and $2.4 \%$ in each year.

\section{Discussion}

This report is the first describing a study of Mycoplasma pneumoniae infection 


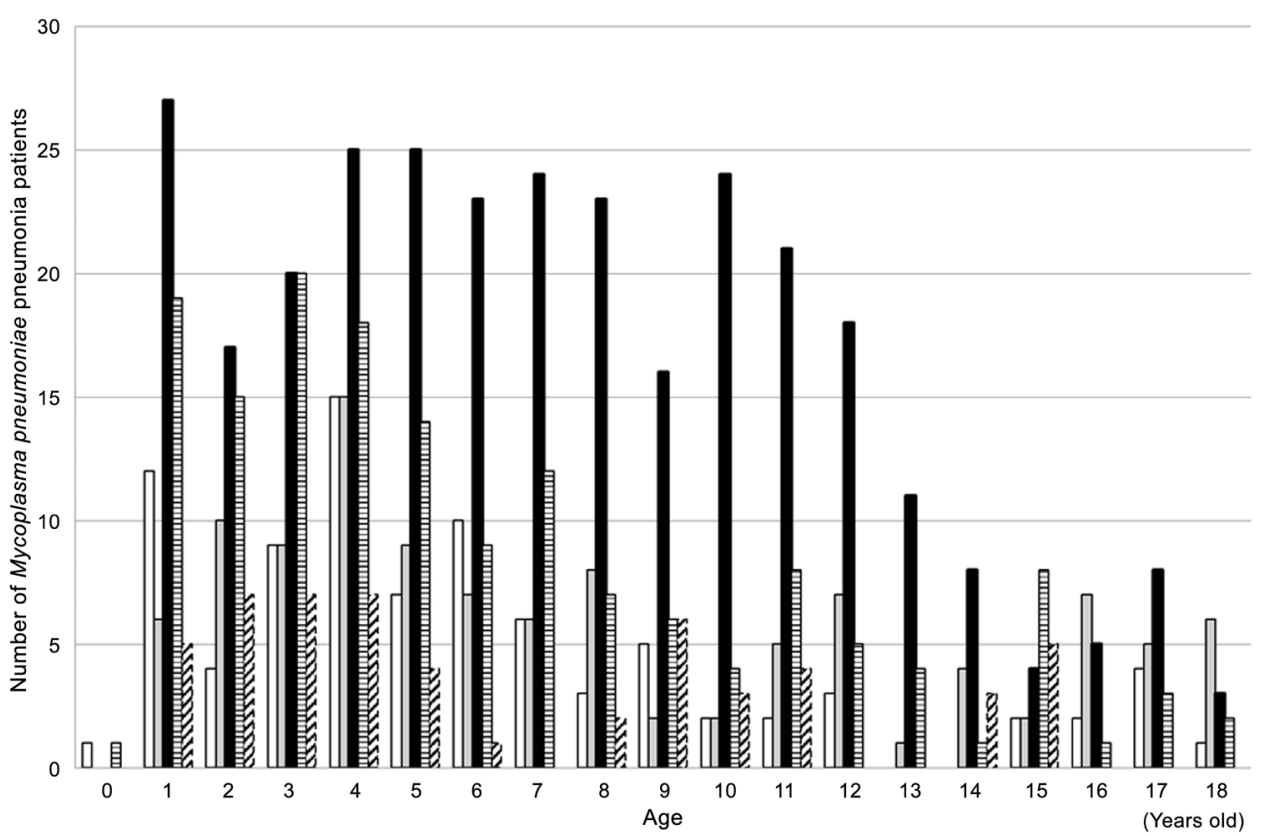

Figure 2. Number of Mycoplasma pneumoniae pneumonia patients by age in Ibaraki prefecture in NOSSID. Note: White bars represent data for the 2010 school year. Gray bars show the 2011 school year. Black bars showthe2012 school year. Bars with - show the 2013 school year. Bars with / show 2014 school year. The school year in Japan is defined as the first April to the end of March of the following year. Children of age 0 - 5 usually attend a nursery school or kindergarten. Age 6 - 11 usually attend an elementary school. Age 12 - 14 usually attend a junior high school. Age 15 17 usually attend a high school.

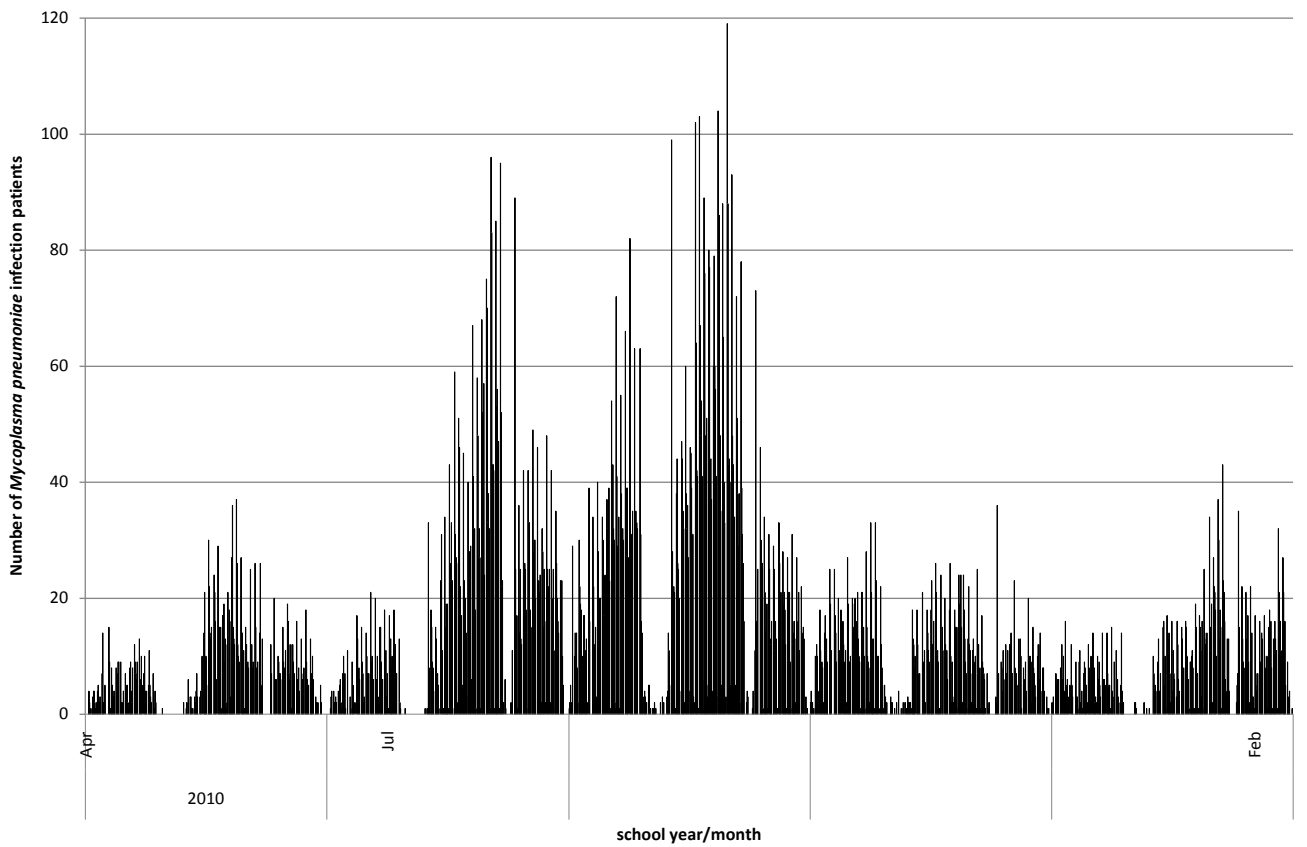

Figure 3. Number of Mycoplasma pneumoniae infection patients in Ibaraki prefecture in (N)SASSy. Note: This figure shows the number of newly diagnosed patients from the 2010 school year to the 2014 school year day by day. School year in Japan is defined as April first to the end of March the following year. Newly diagnosed patients were recorded the first day they absent from (nursery) school, diagnosed as having Mycoplasma pneumoniae infection. 


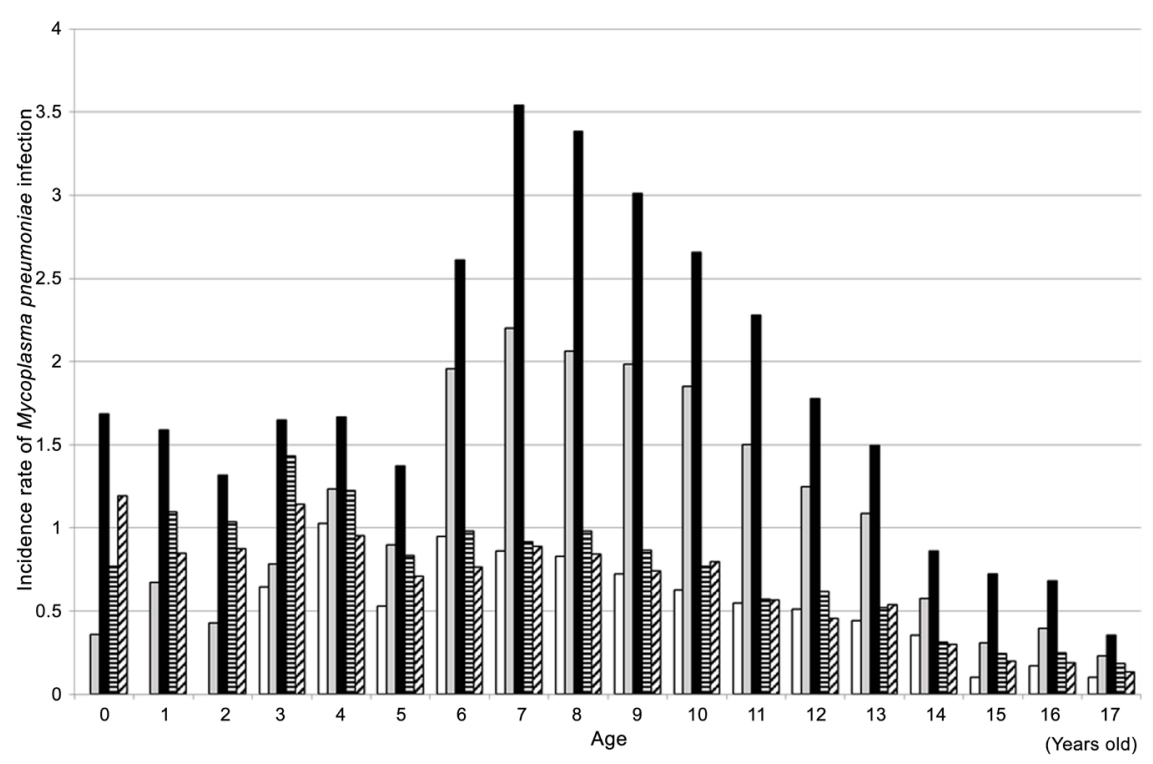

Figure 4. Incidence rate of Mycoplasma pneumoniae infection in Ibaraki prefecture by grade in (N)SASSy. Note: White bars represent the 2010 school year. Gray bars show the 2011 school year. Black bars show the 2012 school year. Bars with - show the 2013 school year. Bar with / show the 2014 school year. The school year in Japan is defined as the first April to the end of March of the following year. Children of age $0-5$ usually attend a nursery school or kindergarten. Age 6 - 11 usually attend an elementary school. Age 12 14 usually attend a junior high school. Age 15 - 17 usually attend a high school.

Table 1. Comparison among of Mycoplasma pneumoniae pneumonia patients in NOSSID and Mycoplasma pneumoniae infection Mycoplasma pneumoniae infection.

\begin{tabular}{cccc}
\hline $\begin{array}{c}\text { School } \\
\text { Year }\end{array}$ & $\begin{array}{c}\text { Number of Mycoplasma } \\
\text { pneumoniae pneumonia } \\
\text { patients in NOSSID }\end{array}$ & $\begin{array}{c}\text { Number of Mycoplasma } \\
\text { pneumoniae pneumonia patients } \\
\text { younger than 18 years old in } \\
\text { NOSSID and its share for all age (\%) }\end{array}$ & $\begin{array}{c}\text { Total number of } \\
\text { infection patients in } \\
\text { (N)SASSy }\end{array}$ \\
\hline 2010 & 264 & $88(33.7)$ & 1809 \\
2011 & 301 & $111(36.9)$ & 4562 \\
2012 & 428 & $302(70.6)$ & 7209 \\
2013 & 186 & $157(84.4)$ & 2552 \\
2014 & 57 & $54(94.7)$ & 2218 \\
\hline
\end{tabular}

Note: Because the school years defined as the first April extending to the end of March of the following year, we define school year in this table as the $14^{\text {th }}$ epidemiological week to the $13^{\text {th }}$ epidemiological week of the following year for NOSSID data.

by age using data of (N)SASSy, a comprehensive survey of students and nursery schoolchildren in Japan. Because NOSSID only receives information of Mycoplasma pneumoniae pneumonia from sentinels, which are $1 \%$ of all medical institutions, it is biased to severe cases that require hospital care. However, some patients with Mycoplasma pneumoniae infection might be moderate without pneumonia and require no hospitalization. Actually, Table 1 presents the NOSSID cover rate to (N)SASSy under 18 years old, which represents pneumonia cases in sentinel over all Mycoplasma pneumoniae infection including mod- 
erate cases without pneumonia: only $2.4 \%$ to $6.2 \%$. As one might expect, these very small proportions reflected both the probability of pneumonia in Mycoplasma pneumoniae infection and the incompleteness of sentinel surveillance in NOSSID: we cannot divide the two effects. At least, we can infer that the NOSSID information might not capture the complete picture of outbreak attributable to Mycoplasma pneumoniae infection. Therefore, we regard the information in (N)SASSy as apparently more suitable for situation awareness of outbreak because of Mycoplasma pneumoniae infection.

Because (N)SASSy represents real-time information collection, evaluation, and sharing among (nursery) schools, public health centers, and physicians, countermeasures using (N)SASSy are expected to be useful for situational awareness of caregivers of children, students, nursery schoolchildren, and the community, interrupting the spread of pathogens and then preventing larger outbreaks. As stated in the last sentence in Results, the information from NOSSID and (N)SASSy is not necessarily the same. In fact, NOSSID includes information only from sentinel hospitals. It is therefore not comprehensive. By contrast, (N)SASSy is comprehensive of hospitals and clinics, but it does not cover patients older than 18 years old. It is noteworthy that because NOSSID has some information of patients older than 18 years old, NOSSID and (N)SASSy are mutually complementary. Therefore, it is quite important to monitor both at the same time.

Rapid diagnosis testing of Mycoplasma pneumoniae was approved for Japan in August 2013 [9]. In general, approval and reimbursement of rapid tests by health insurance increases the number of diagnoses because of economic incentives to administer this test. However, as shown in Figure 1 and Figure 2, the incidence after approval was much lower than before. Therefore, our findings show high activity in 2012 and show that it was unaffected by introduction of the rapid test.

We have some limitations that are applicable to this study. First, (N)SASSy does not cover children who do not attend nursery schools or kindergartens, or workers younger than 18 years old. Especially, $63.8 \%$ of children younger than three years old do not go to a nursery school or kindergarten [10]. If some outbreak occurs among them, then (N)SASSy's information might be under-biased. However, its probability is apparently quite lower than children who attend nursery schools. Moreover, we consider that outbreaks among nursery schoolchildren can reflect outbreaks among children who do not go to nursery schools.

A second limitation is that the definitions of ages in NOSSID and (N)SASSy are not the same. In (N)SASSy, the age definition is grade level in (nursery) schools, but NOSSID uses the actual age of the patients, which might lead to some discrepancy.

A third limitation is that (N)SASSy cannot cover adults, including workers who are younger than eighteen years old. Some infectious diseases, including meningococcal meningitis, are well known to lead to outbreaks in dormitories for adults. 


\section{Conclusion}

Mycoplasma pneumoniae infection is a common pediatric infectious disease. Some patients rarely develop severe infections. Therefore, wider outbreaks must be prevented. (N)SASSy can provide timely intervention at the initial phase of outbreak by monitoring situations in (nursery) schools and by comparing data to baseline information.

\section{Acknowledgements}

This research was benefited by a Grant-in-Aid for Scientific Research (C) $15 \mathrm{~K} 01676$ "Research for guidance and effort for introduction of (nursery) school absenteeism surveillance system" headed by Dr. Tamie Sugawara, Senior Scientist, National Institute of Infectious Diseases.

\section{Conflicts of Interest}

The authors declare no conflicts of interest regarding the publication of this paper.

\section{References}

[1] Powell, D.A. (2004) Mycoplasmal Infections. In: Behrman, R.E., Kliegman, R.M. and Jenson, H.B., Eds., Nelson Textbook of Pediatrics Website, 17th Edition, Elsevier, Saunders, 990-992.

[2] National Institution of Infectious Diseases (2007) Mycoplasmal Pneumonia as of 2006, Japan. IASR, 28, 31-32. http://idsc.nih.go.jp/iasr/28/324/tpc324.html

[3] National Institute of Infectious Diseases (2012) Mycoplasmal Pneumonia as of September 2012, Japan. IASR, 33, 261-262. https://www.niid.go.jp/niid/en/iasr-vol33-e/865-iasr/2738-tpc392.html

[4] Sugiura, H., Hata, T., Kodama, K., Oikawa, K., Imamura, T., Ohkusa, Y. and Okabe, N. (2010) Examination of the Effectiveness of Class Closures for A/H1N1 (2009) pdm Using the Automatic Information Sharing System for School Absentees. Japanese Journal of School Health, 52, 214-218. (In Japanese)

[5] Ohkusa, Y., Sugawara, T., Mitani, M., Sugiura, H. and Okabe, N. (2011) Development and Evaluation of a School Absenteeism Reporting System. Japanese Journal of School Health, 53, 312-319. (In Japanese)

[6] Watanabe, M., Kurita, J., Takagi, T., Nagata, N., Nagasu, N., Sugawara, T., et al. (2016) Early Detection and Response for Measles and Rubella Cases through the (Nursery) School Absenteeism Surveillance System in Ibaraki Prefecture. Japanese Journal of Public Health, 63, 209-214.

[7] Yamakawa, M., Yamauchi, M., Nidaira, M., Azuma, T., Nakasone, T., Ando, F., et al. (2017) Enhanced Public Health Surveillance for the Sixth Worldwide Uchinanchu Festival Conducted by the Okinawa Prefectural Government, Japan. Journal of Biosciences and Medicines, 5, 106-115. https://doi.org/10.4236/jbm.2017.59010

[8] Takagi, T., Kawamata, R., Kawamata, Y., Wada, C. and Yamamoto, K. (2014) Detection of Mycoplasma Pneumonia P1 Gene by Polymerase Chain Reaction (PCR) and Macrolide Resistant Gene Sequence by DNA Sequencer. Annual Report of Ibaraki Prefectural Institute of Public Health, 52, 31-34. 
[9] Miyashita, N., Kawai, Y., Yamaguchi, T., Ouchi, K., Oka, M. and Atypical Pathogen Study Group (2011) Clinical Potential of Diagnostic Methods for the Rapid Diagnosis of Mycoplasma pneumoniae Pneumonia in Adults. European Journal of Clinical Microbiology \& Infectious Diseases, 30, 439-446. https://doi.org/10.1007/s10096-010-1107-8

[10] Ministry of Health, Labor and Welfare (2009) Nationwide Survey on Families and Children. Ministry of Health, Labor and Welfare, Tokyo. 